\title{
ARTICLE Chemogenetic activation of ventral tegmental area GABA neurons, but not mesoaccumbal GABA terminals, disrupts responding to reward-predictive cues
}

\author{
Ken T. Wakabayashi ${ }^{1,2}$, Malte Feja ${ }^{1}$, Ajay N. Baindur ${ }^{1}$, Michael J. Bruno ${ }^{1}$, Rohan V. Bhimani ${ }^{3}$, Jinwoo Park ${ }^{3}$, Kathryn Hausknecht iD $^{2}$, \\ Roh-Yu Shen ${ }^{2}$, Samir Haj-Dahmane ${ }^{2}$ and Caroline E. Bass (iD)
}

Cues predicting rewards can gain motivational properties and initiate reward-seeking behaviors. Dopamine projections from the ventral tegmental area (VTA) to the nucleus accumbens (NAc) are critical in regulating cue-motivated responding. Although, approximately one third of mesoaccumbal projection neurons are GABAergic, it is unclear how this population influences motivational processes and cue processing. This is largely due to our inability to pharmacologically probe circuit level contributions of VTA-GABA, which arises from diverse sources, including multiple GABA afferents, interneurons, and projection neurons. Here we used a combinatorial viral vector approach to restrict activating Designer Receptors Exclusively Activated by Designer Drugs (DREADDs) to GABA neurons in the VTA of wild-type rats trained to respond during a distinct audiovisual cue for sucrose. We measured different aspects of motivation for the cue or primary reinforcer, while chemogenetically activating either the VTA-GABA neurons or their projections to the NAc. Activation of VTA-GABA neurons decreased cue-induced responding and accuracy, while increasing latencies to respond to the cue and obtain the reward. Perseverative and spontaneous responses decreased, yet the rats persisted in entering the reward cup when the cue and reward were absent. However, activation of the VTA-GABA terminals in the accumbens had no effect on any of these behaviors. Together, we demonstrate that VTA-GABA neuron activity preferentially attenuates the ability of cues to trigger reward-seeking, while some aspects of the motivation for the reward itself are preserved. Additionally, the dense VTA-GABA projections to the NAc do not influence the motivational salience of the cue.

Neuropsychopharmacology (2019) 44:372-380; https://doi.org/10.1038/s41386-018-0097-6

\section{INTRODUCTION}

When neutral cues are repeatedly paired with a reward, they can become powerful incentives for reward-seeking, a process that is heavily dependent upon mesoaccumbal dopamine (DA) [1-3]. Yet, $Y$-aminobutyric acid (GABA) neurons are also present in the ventral tegmental area (VTA), and their role in regulating VTA mediated reward processes is not well understood. Pharmacological studies show that intra-VTA microinfusions of GABA agonists attenuate reward-predictive cue behavior, cue-evoked firing of target neurons in the nucleus accumbens (NAC), and disrupt both sucrose self-administration and feeding [4-6]. However, pre- and post-synaptic GABA receptors are ubiquitous throughout the VTA $[7,8]$, which also receives numerous GABA afferents from the NAC $[9-11]$, the pedunculopontine tegmental nucleus, and the lateral hypothalamus ([8], [12] [13]), making it unclear if these pharmacological effects are attributable to resident GABA neurons within the VTA, signaling directly through GABA receptors on DA neurons, or by modulating GABA and/or glutamate afferents.

VTA-GABA neurons are thought to consist primarily of GABA interneurons, while an estimated $20 \%$ project to other areas, including the NAc $[7,14,15]$. In fact, approximately one third of mesoaccumbal projecting neurons are GABAergic [16]. These unique GABA projection neurons likely have distinct influences on reward-seeking behaviors, however compared to DA, relatively little is known about the functional role of either VTA-GABA neurons or the mesoaccumbal GABA projections in reward-cue processing.

Recent advances in genetics have permitted the direct examination and manipulation of VTA-GABA neurons in mice, however behavioral assessments on cue processing have produced mixed results. For example, VTA-GABA neurons displayed enhanced firing in response to a reward-predictive odor cue [17]. Yet, while one study demonstrated that optogenetic activation of VTA-GABA cell bodies disrupted anticipatory licking to a sucrosepredictive odor [18], another failed to show an effect on anticipatory licking induced by a predictive light-tone cue [19]. In an appetitive Pavlovian conditioning paradigm, inhibition of VTA-GABA neurons tended to delay task acquisition [13].

Studies employing optogenetic stimulation of VTA-GABA terminals in the NAc are sparse but have likewise produced confounding results. In one study, activation of these GABA terminals had no effect on sucrose consumption [19]. Yet another study showed that activation of VTA-GABA terminals in the NAC during training enhanced discrimination learning,

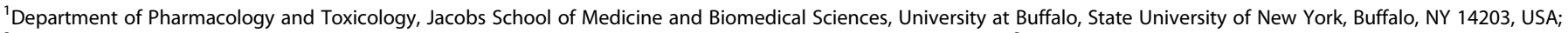

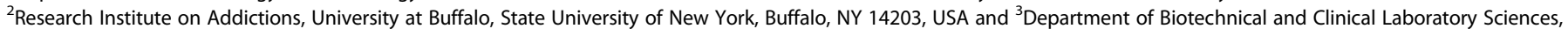
University at Buffalo, State University of New York, Buffalo, NY 14214, USA
}

Correspondence: Caroline E. Bass (cebass@buffalo.edu)

Received: 21 August 2017 Revised: 3 May 2018 Accepted: 5 May 2018

Published online: 22 May 2018 
such that in later testing the mice were able to discriminate between a cue predicting an aversive stimulus and a similar but unpaired cue [20]. These studies indicate that either the mesoaccumbal GABA projections influence only negative reinforcement, or that the anticipatory licking behavior could not capture the effects of activating these projections on reward-predictive cues.

In this study, we sought to determine how VTA-GABA neurons influence instrumental responding to reward-predictive cues in a rat Pavlovian instrumental task, and whether mesoaccumbal GABA projections also contribute to these behaviors. Targeted activation of VTA-GABA neurons was achieved using a novel combinatorial viral approach to deliver activating DREADDs (Designer Receptors Exclusively Activated by Designer Drugs, hM3D) to VTA-GABA neurons in wild-type rats. This circuit level approach overcomes the promiscuity of locally delivered pharmacological agents. Further, our sophisticated operant model is sensitive to GABAergic pharmacological manipulations [6], and requires a robust behavioral response which integrates multiple reward-related processes, including attending to a predictive cue, seeking out the reward, and consuming it. Thus, our approach provides a better understanding of how VTA-GABA neurons and mesoaccumbal GABA projections influence cue motivated responding and definitely determine if these VTA-GABA neurons influence responding to reward-predictive cues.

\section{METHODS AND MATERIALS}

\section{Subjects}

Long-Evans rats (Envigo, Indianapolis, IN) weighing $\sim 290-320 \mathrm{~g}$ were individually housed under a $12 \mathrm{~h} / 12 \mathrm{~h}$ light/dark cycle (lights on at 3 PM), with all behavioral experiments conducted at 9 AM. Food and water were available ad libitum. Male Sprague Dawley rats $(200-300 \mathrm{~g})$ were used for electrophysiological experiments. All procedures complied with the Guide for the Care and Use of Laboratory Animals (NIH, Publication 865-23) and were approved by the Institutional Care and Use Committee at the University at Buffalo.

\section{Adeno-associated virus (AAV)}

To target GABA neurons, we used a combinatorial viral vector approach in which Cre recombinase expression is driven by the rat glutamate decarboxylase 1 (GAD1) promoter [21], while the excitatory DREADD (hM3D) or channelrhodopsin-2 (ChR2) was provided in a Cre dependent DIO (Double-floxed Inverse Orientation) configuration, which requires Cre recombinase to flip it in line with the generalized EF1a promoter. Specifically, DIOChR2-EYFP-AAV2/10 (electrophysiology) or DIO-hM3D-mCherryAAV2/10 (electrophysiology and behavior) were co-infused with GAD1-Cre-AAV2/10 into the VTA (Fig. 1a). Previously we used this combinatorial approach to restrict ChR2 to tyrosine hydroxylase positive neurons in the VTA [22-24].

Stereotaxic virus injection surgery

Rats $(n=23)$ were bilaterally infused with either $1 \mu$ l of virus (333 $\mathrm{nl}$ of GAD1-Cre co-infused with either $667 \mathrm{nl}$ of DIO-hM3DmCherry or DIO-ChR2-EYFP) or $0.5 \mu$ of virus $(167 \mathrm{nl}$ of GAD1-Cre co-infused with $333 \mathrm{nl}$ of DIO-hM3D-mCherry) into the VTA (in $\mathrm{mm}$ from bregma: $\mathrm{AP}-5.6, \mathrm{ML} \pm 1.0, \mathrm{DV}-7.8$ ) over 2 min using a Drummond Nanoject injector equipped with a glass capillary, and 8 of these were then implanted with double 26 ga cannulae (PlasticsOne, Roanoke, VA) in the NAc (in mm from bregma: AP, $+1.5, \mathrm{ML}, \pm 1.1, \mathrm{DV},-5.5)$ in a procedure detailed elsewhere [25]. Rats trained on the incentive cue (IC) task were allowed a minimum of 5 days before resuming behavioral sessions, and all rats were allowed $>2$ weeks before undergoing their respective drug treatments or procedures. Additional rats $(n=15)$ were infused with GFP control virus to serve as sham controls for various behavioral measures and electrophysiological experiments; of these 6 were also implanted with cannulae in the NAC as described above.

Drugs and treatment

For activation of VTA-GABA cell bodies, clozapine $\mathrm{N}$-oxide (CNO, National Institutes of Drug Abuse Drug Supply Program, Baltimore, MD) was dissolved with $0.9 \%$ sterile saline and $0.3 \mathrm{mg} / \mathrm{kg}$ administered intraperitoneally (i.p.) $30 \mathrm{~min}$ prior to the session $[26,27]$. CNO and saline injections were randomized and occurred at least 3 days apart. To selectively activate VTA-GABA terminals in the NAc, rats implanted with cannula were bilaterally infused with $0.3 \mu \mathrm{l}$ of saline or $1 \mathrm{mM}$ CNO $(300 \mu \mathrm{mol}$ total) dissolved in $0.9 \%$ sterile saline [28] at $0.15 \mu \mathrm{l} / \mathrm{min}$ in a procedure adapted from others [25] and detailed further in Supplementary materials and methods. In order to rule out a potential role of clozapine on behavioral responses, clozapine (CZP, Enzo Life Sciences, Inc., Farmingdale, NY) was dissolved with $0.9 \%$ sterile saline and 0.3 $\mathrm{mg} / \mathrm{kg}$ administered i.p. $30 \mathrm{~min}$ prior to the session.

\section{Behavioral apparatus}

Testing occurred in operant chambers (Med-Associates, Georgia, VT) within a sound-attenuating cubicle, equipped with two illuminated infrared nosepoke ports surrounding a centrally located liquid receptacle equipped with an infrared entry detector. A white houselight and a tone generator speaker were located on the opposite wall. Each chamber was equipped with a pump to deliver $10 \%$ sucrose. Locomotor testing occurred in open-field activity monitors (Med-Associates, Georgia, VT) within a soundattenuating cubicle. A white houselight was located in the corner of the cubicle and a ventilation fan provided white noise.

Training and behavioral paradigm

IC task training. The IC task is modified from others described elsewhere $[6,25,29]$ and in detail in Supplementary methods.

Free drinking. A free drinking session was conducted in the operant chambers, where head entry into the reward receptacle activated the pump, delivering sucrose continuously into the cup until the rat left the receptacle. The first 2-min was analyzed as the total volume consumed in this period was equivalent to that consumed after saline pretreatment during the IC sessions. Chambers were checked after each session to confirm all the liquid had been consumed. Head entries in the previously active or inactive nosepoke port had no programmed consequences.

Locomotor activity. Activity was assessed in a subset of rats (hM3D, $n=7$; GFP, $n=6$ ) in open-field chambers for 20 min. Rats were acclimatized to the chambers for two consecutive days, and pretreatment with $\mathrm{CNO}$ or saline 30 min prior to the session on the third day.

Histological verification

Histological verification of virus and cannula placements were conducted for all subjects using immunohistochemistry, as detailed in Supplementary methods, and described elsewhere [22].

Electrophysiology and LED photostimulation

Neurons in the VTA were visualized using an Olympus BX-51-WI fluorescent microscope. Somatic recordings were obtained from EYFP negative putative DA neurons $(n=4)$ with patch electrodes (3-5 M 2 ) filled with a solution containing (in $\mathrm{mM}$ ): 120 cesium methanesulfonic acid; Cs-Cl; $10 \mathrm{Na}_{2}$-phosphocreatine, $10 \mathrm{HEPES} ; 1$ $\mathrm{MgCl}_{2} ; 1$ EGTA; $2 \mathrm{Na}_{2}$-ATP; $0.25 \mathrm{Na}-\mathrm{GTP}$ (pH = 7.3; adjusted with Cs$\mathrm{OH}$; Osmolarity: 280 to $290 \mathrm{mOsmol} / \mathrm{l}$ ) or a solution containing: 120 potassium gluconate, $10 \mathrm{Na}_{2}$-phosphocreatine, $10 \mathrm{KCl}, 10 \mathrm{HEPES}, 1$ $\mathrm{MgCl}_{2}, 1 \mathrm{EGTA}, 2 \mathrm{Na}_{2}-\mathrm{ATP}$, and $0.25 \mathrm{Na}-\mathrm{GTP}(\mathrm{pH}=7.3)$. All recordings 
A

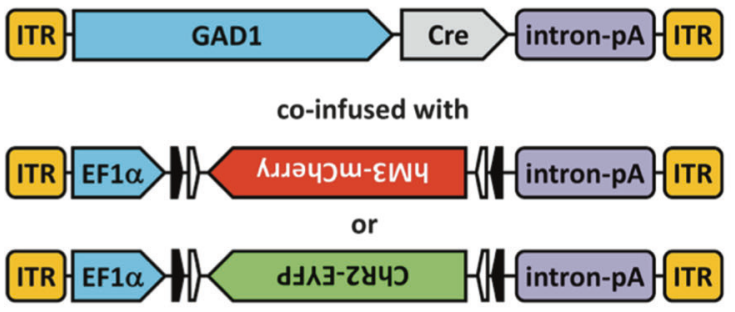

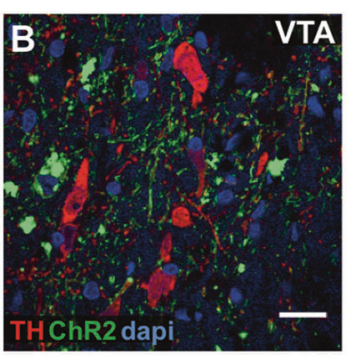

$\mathbf{F}$
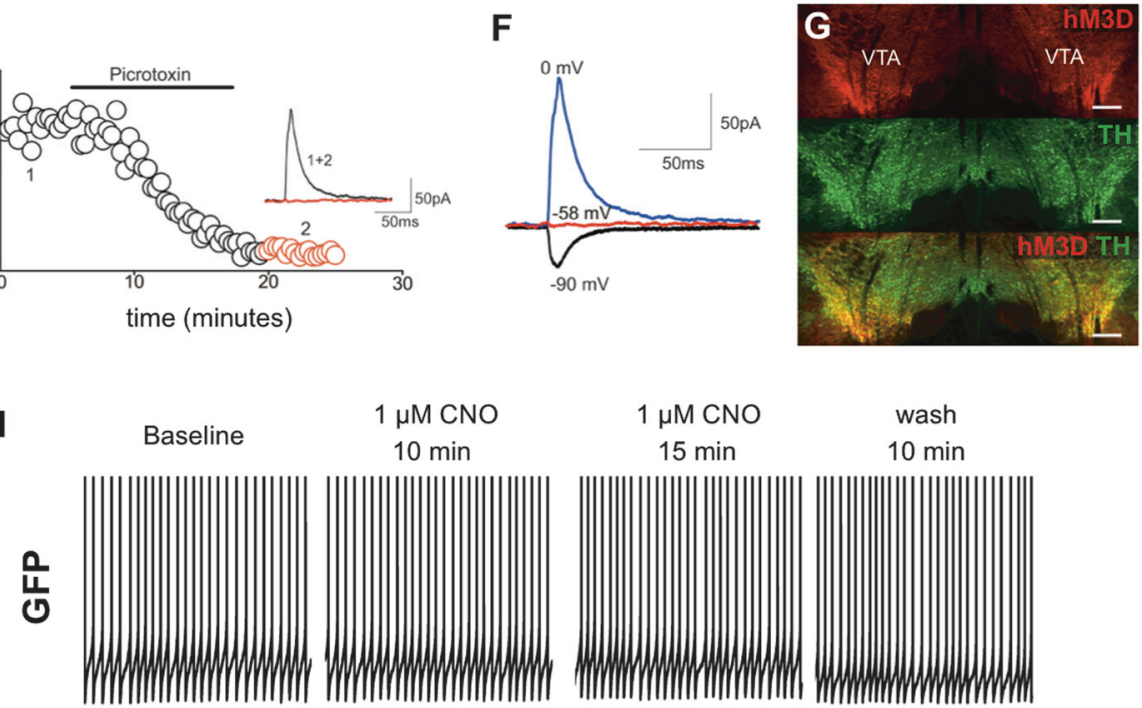

wash

$10 \mathrm{~min}$
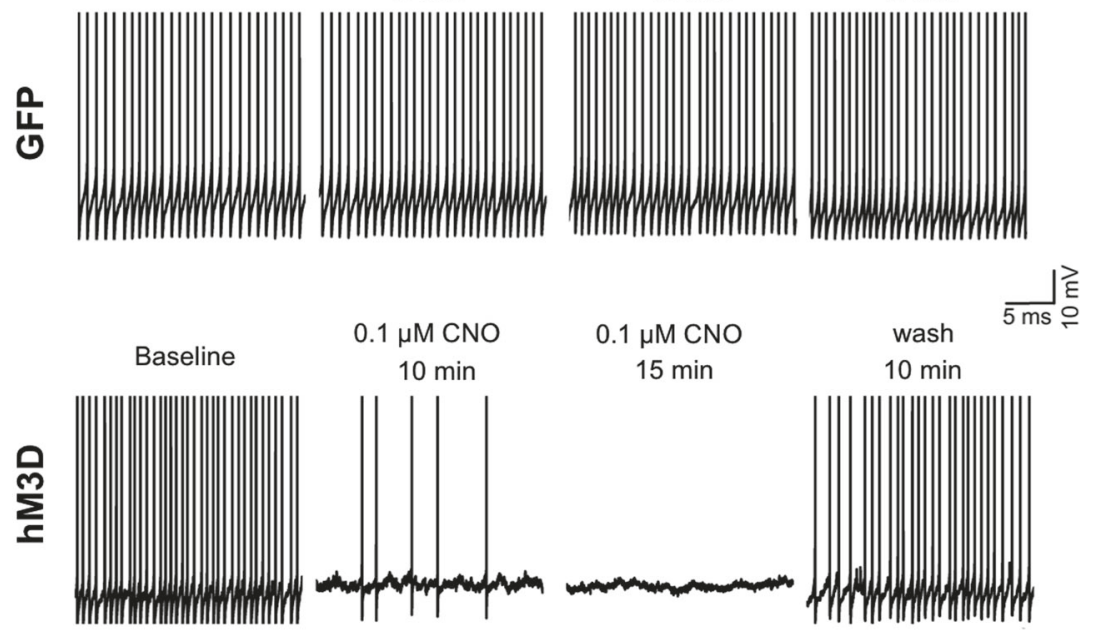

Fig. 1 Combinatorial adeno-associated virus (AAV) targeting of GABA neurons in the VTA. a Viral constructs used in this study, white and black arrow blocks indicate the lox sites constituting the DIO configuration. b Double immunofluorescence staining for tyrosine hydroxylase (TH; red) and channelrhodopsin-2-enhanced yellow fluorescent protein (ChR2-EYFP; green) in the VTA, scale bar indicates $20 \mu \mathrm{m}$, and (c) proportion of dopamine (DA) and non-DA neurons expressing ChR2 (406 neurons counted, $n=4$ ). d ChR2-EYFP terminals in the NAc, scale bar indicates $20 \mu \mathrm{m}$. e lllustrates optical post synaptic current (oPSC) induced by photostimulation of ChR2 in the VTA and recorded from nearby putative DA neuron voltage clamped at $-90 \mathrm{mV},-58 \mathrm{mV}$, and $0 \mathrm{mV}(n=4$, mean of 10 trials at $0.05 \mathrm{~Hz})$. The GABA $\mathrm{A}_{\mathrm{A}}$ antagonist picrotoxin (100 $\mu \mathrm{M}$ ) decreases the amplitude of oPSCs. $\mathbf{f}$ oPSCs reverse polarity at $\mathrm{E}_{\mathrm{cl}}$ (i.e., $-58 \mathrm{mV}$ ). g Representative double fluorescent image of GAD1 targeted hM3D (top), TH (middle) and merged (bottom) of the left and right VTA, scale bar indicates $100 \mu \mathrm{m}$. $\mathbf{h}$ Double immunofluorescence staining for tyrosine hydroxylase (TH; cyan) and hM3D-mCherry (magenta) in the VTA, scale bar indicates $20 \mu \mathrm{m}$. i Illustrates representative recording traces from spontaneously firing DA neurons in a GFP sham rat (top) and a GAD1 directed hM3D rat (bottom), respectively. At $1 \mu \mathrm{M}$, $\mathrm{CNO}$ had no effects on the spontaneous firing of a DA neuron recorded from a GFP sham rat. In contrast, CNO at $0.1 \mu \mathrm{M}$ completely inhibited the spontaneous firing of a DA neuron recorded from a GAD1 directed hM3D rat. $\mathbf{j}$ Diagram of events for a successful response during the IC task denoting the rat snout (gray cone) responding to the IC $(A)$, by nosepoking $(B)$, followed by a reward cup entry $(C)$

were performed in the presence of 6,7-dinitroquinoxaline-2,3-dione (DNQX; $20 \mu \mathrm{M}), \mathrm{D}-(-)-2-$ Amino-5-phosphonopentanoic acid (D-APV; $20 \mu \mathrm{M})$ to block AMPA (a-amino-3-hydroxy-5-methyl-4-isoxazolepropionic acid) and NMDA (N-Methyl-D-aspartic acid) receptors, respectively. LED photostimulation was delivered using a Prizmatix LED module $(465 \mathrm{~nm})$. Light was delivered through $200-\mu \mathrm{m}$ path optical cable with a bare $1 \mathrm{~cm}$ glass exposed. Optically induced currents were triggered at a frequency of $0.005 \mathrm{~Hz}$ with light pulses (duration: $5 \mathrm{~ms}$, intensity: $2-5 \mathrm{~mW}$ ). For chemogenetic experiments,
CNO was applied at concentrations of $0.1,0.3$, or $1.0 \mu \mathrm{M}$ for $15-20$ min followed by a wash out period.

Measures and data analysis

We quantified four basic metrics in the incentive cue task: the response ratio for the IC predicting availability of sucrose (\#nosepokes resulting in a reward/\#ICs), the nosepoke latency (time; $T)$ to emit a rewarded nosepoke $\left(T_{\text {nosepoke }}-T_{I C}\right)$, the reward cup latency to enter the cup after a rewarded nosepoke ( $T_{\text {receptacle }}{ }^{-}$ 


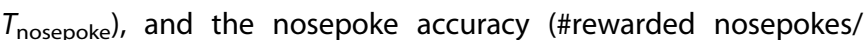
\#total nosepokes). We also counted the number of ICs before the rats earned their first reward. We classified unrewarded active nosepokes as "perseverative" if they occurred while the reward was being delivered (during the CS + and pump on), "near misses" if they occurred $0-8 \mathrm{~s}$ after an unrewarded IC, or "spontaneous" if they occurred in the variable interval between IC presentations. Overall reward-cup behavior was quantified as the total cup entries per reward earned. To characterize common action patterns associated in response to an IC during the task, we quantified the number of times rats engaged in rewarded sequences: a successful nosepoke during an IC presentation followed by entry into the reward receptacle (IC $\left(A_{+}\right) \rightarrow$ nosepoke $(B) \rightarrow$ reward cup $(C))$, or a reward cup entry prior to a successful nosepoke response during an IC $\left(A_{+} \rightarrow C \rightarrow B \rightarrow C\right)$. Three unrewarded action patterns were quantified: entering the reward receptacle only during an IC $\left(\mathrm{A}_{+} \rightarrow \mathrm{C}\right)$, ignoring the IC and then entering the reward cup when the IC was off (i.e., $A_{0} \rightarrow C$ ), and when the rat did not respond during or between the IC presentations $\left(A_{0}\right)$. The total volume consumed during a freedrinking session was derived from the length of time the rat remained inside the reward receptacle and the flowrate of the pump. Locomotion was quantified as distance traveled in the session.

Statistical comparisons were conducted using $t$-tests, Wilcoxon matched-pairs signed-rank tests, One-way, Two-way repeatedmeasures (RM), and mixed design ANOVA, followed by a HolmsSidak correction for pairwise comparisons, where appropriate. Normality was tested with a Shapiro-Wilk test. Significance was set to $a=0.05$. Additional details on the statistical tests are provided in the relevant results or figure legends.

\section{RESULTS}

To validate the dual virus targeting system, we first co-infused the EF1a-DIO-ChR2-EYFP-AAV2/10 virus with the GAD1-Cre-AAV2/10 virus into the VTA. Double immunofluorescence for EYFP and TH revealed that ChR2-EYFP expression occurred primarily in non-DA neurons (Fig. 1b) in reported proportions for DA and GABA neurons in the VTA $(\sim 74 \%$ of total neurons were TH+/ChR2-, $\sim 25 \% \mathrm{TH}-/$ ChR2 + , and $<0.5 \% \mathrm{TH}+/ \mathrm{ChR2}+, 406$ total, $n=4$, Fig. 1c, [30, 31]). Numerous ChR2-EYFP + terminals were also observed in the NAc (Fig. 1d). To verify that the ChR2+ neurons were GABAergic, we examined whether photoactivation of ChR2 triggers GABA-mediated synaptic currents in putative DA neurons. We found that in the presence of AMPA and NMDA receptor antagonists, local photostimulation elicited robust optical inhibitory post-synaptic currents (oIPSCs) in putative DA neurons (Fig. 1e, f). olPSCs were recorded at various holding potentials (Fig. 1f, each trace is the average of 10 trials at $0.05 \mathrm{~Hz}$ ). These oIPSCs reversed polarity at the equilibrium potential of chloride ions (i.e., $-58 \mathrm{mV}$ ) determined in our experimental condition and were blocked by the $\mathrm{GABA}_{\mathrm{A}}$ receptor antagonist picrotoxin $(100 \mu \mathrm{M}$, Fig. $1 \mathrm{e}$, inset is an example oIPSC collected before and during picrotoxin application). Collectively, this experiment indicates that $\mathrm{ChR2}+$ neurons in the VTA are indeed GABAergic and their activation induces a robust GABA-IPSC in putative VTA-DA neurons. Additional rats were co-infused with EF1aDIO-hM3D-mCherry-AAV2/10 and GAD1-Cre-AAV2/10 viruses into the VTA (Fig. 1a). Robust and widespread hM3D-mCherry expression was observed in the VTA (Fig. 1g). Note that although some yellow is observed in the bottom panel, the objective used to generate this image cannot resolve closely located signals. Importantly, as in Fig. 1b, hM3D-mCherry+ neurons did not co-localize with $\mathrm{TH}+$ neurons (Fig. 1h). Additional recordings were performed to test CNO effects. We showed that bath applied CNO at $10 \mu \mathrm{M}$ had no effects on the spontaneous firing of a DA neuron recorded from a GFP sham control rat (Fig. 1i, top panel) while the same concentration of CNO completely inhibited neuron firing in a DA neuron which recovered after wash out. In addition, the spontaneous firing of DA neurons was completely inhibited at lower CNO concentrations (0.1, 0.3 , and $1 \mu \mathrm{M} ; n=3$; Fig. $1 \mathrm{i}$, bottom panel). In all of these neurons, spontaneous firing returned upon wash out of CNO. These viruses were then applied to a new cohort of rats, which were trained on the IC task (Fig. 1j, Supplementary methods).
A
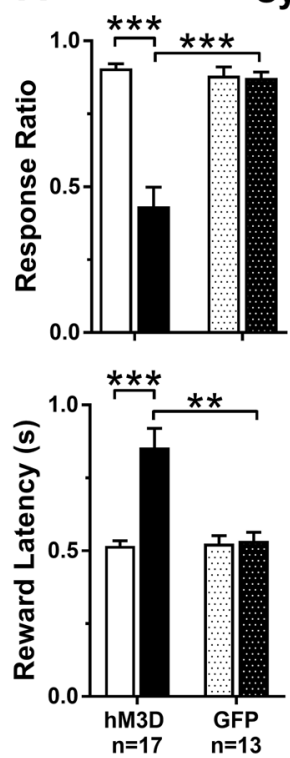

Systemic
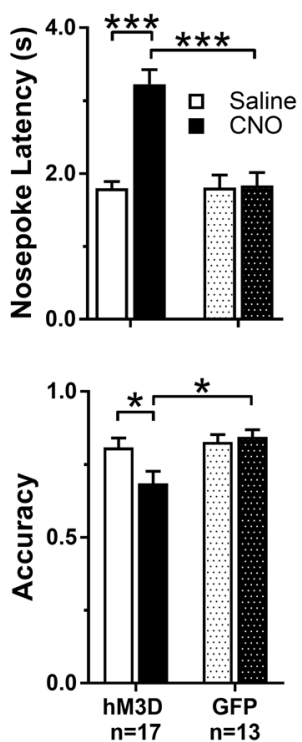

B
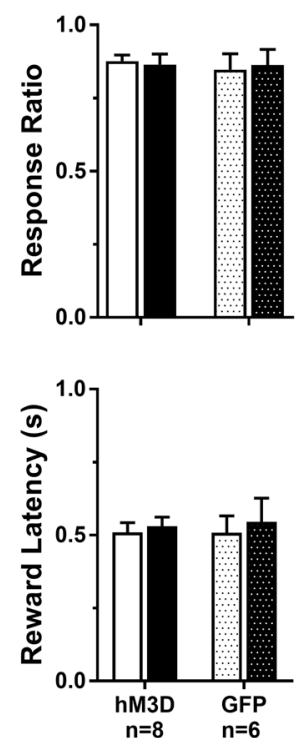

Intra-NAc
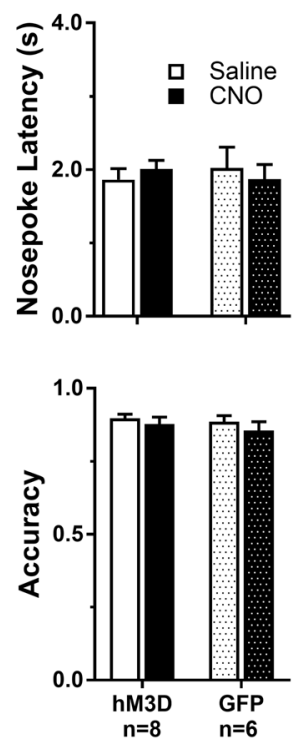

Fig. 2 Activation of VTA-GABA neurons regulate incentive cue (IC) task performance. All data are shown as mean \pm SEM. a Response ratio and accuracy significantly decreased, and latency to nosepoke and enter reward cup significantly increased after systemic CNO in rats expressing hM3D compared to saline and rats expressing GFP. $\mathbf{b}$ Intra-NAc injections of CNO had no effect on IC performance across the behavioral metrics, in either hM3D or GFP expressing rats. Asterisks represent significant differences determined by a Holms-Sidak post hoc test $\left({ }^{*} p<\right.$ $0.05,{ }^{* *} p<0.01,{ }^{* * *} p<0.001$ ) 
A
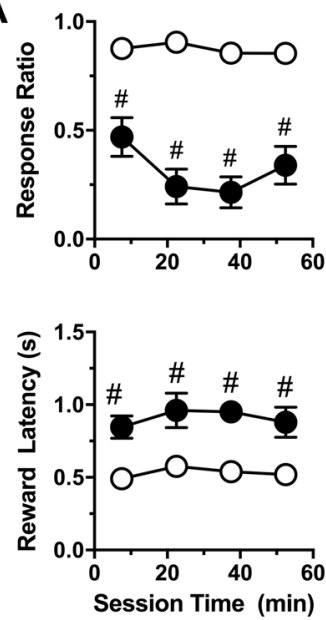

B

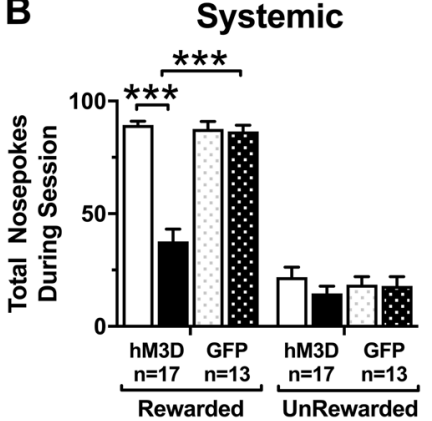

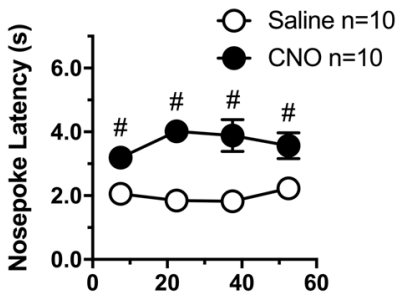

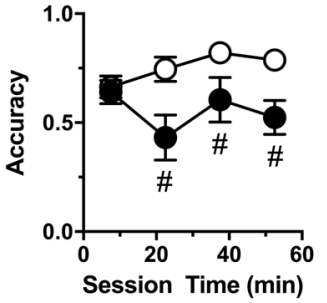

Fig. 3 VTA-GABA neuron mediated decreases in incentive cue (IC) task performance are not mediated by inappropriate nosepoking. All data are presented as mean \pm SEM. a Systemic CNO significantly decreased the response ratio and accuracy throughout the 1-h session when compared to saline control while the latency to nosepoke after the presentation of an IC and enter the reward cup significantly increased. b The total number of rewarded and unrewarded nosepokes during a session after systemic and intraNAc CNO administration. The number of rewarded nosepokes significantly decreased after systemic but not intra-NAc CNO pretreatment compared to saline and GFP controls. For clarity, we report all significant differences in (a) as $\# p<0.0001$. Asterisks represent significant differences between saline and CNO determined by a Holms-Sidak post hoc test in all other panels ${ }^{* * *} p<$ 0.001)

Effect of VTA-GABA stimulation on performance in the incentive cue task

We initially assessed the effects of systemic and intra-NAc CNO administration on overall IC task performance during the entire 1$h$ session in rats expressing either hM3D or sham GFP controls (Fig. 2). When pretreated systemically by i.p. CNO (Fig. 2a), there was a significant Treatment $\times$ Virus interaction, decreasing mean response ratios $\left(F_{1,28}=48.02, p<0.0001\right)$ and accuracy $\left(F_{1,28}=\right.$ 9.39, $p=0.005)$ while increasing nosepoke $\left(F_{1,28}=45.05, p<\right.$ $0.0001)$ and reward $\left(F_{1,28}=15.60, p<0.0001\right)$ latencies only in rats expressing hM3D. Of note, CNO itself did not have a behavioral effect in any of the IC task metrics in rats infused with a sham GFP virus. Activation of GABA terminals in the NAC alone by intra-NAC CNO infusions had no effect on any of the performance metrics in either rats expressing hM3D or sham GFP controls (Fig. 2b). Importantly, this dose of microinfused CNO is effective in other studies ([28], unpublished results).

We further examined the effect of systemic VTA-GABA chemogenetic activation on task performance in a subset of rats

(Fig. 3a). Rats maintained a stable baseline of responding over the 1-h session after saline pretreatment, and did not differ significantly from GFP controls pretreated with saline or CNO (Figure S1A). However, when pretreated with CNO i.p., rats showed a significant decrease in response ratio compared to saline performance (Two-way RM ANOVA, main effect of Time $F_{3,27}=5.58, p=0.0041$, treatment, $p<0.0001, F_{1,9}=6.18$, Time $\times$ Treatment $F_{3,27}=5.51, p=0.0044$ ) and overall accuracy (main effect of Treatment, $F_{1,9}=6.41, p=0.0321$ ). Rats also uniformly increased their latency to nosepoke (main effect of Treatment $F_{1,9}$ 69.2, $p<0.0001$ ), and obtain the reward (main effect of Treatment $\left.F_{1,9}=29.8, p=0.0004\right)$. All other effects and interactions were not significant.

We next examined the type of nosepokes emitted (Fig. 3b) to determine whether changes in accuracy after VTA-GABA stimulation (Fig. 3a) result from either decreased responding to the IC or increases in inappropriate nosepoking between ICs. In systemically treated rats, there was a significant Treatment $\times$ Virus interaction (Fig. 3b, $F_{1,57}=33.27, p<0.0001$ ) and a significant Treatment $\times$ Nosepoke type interaction $\left(F_{1,57}=26.64, p<0.0001\right)$ indicating that VTA-GABA neuron activation decreased rewarded nosepokes in rats expressing the DREADD but not GFP controls. Furthermore, there was no effect of stimulating the NAc projections directly (Fig. 3b). We next categorized the nosepokes as spontaneous (occurring between IC), perseverative (multiple nosepokes during IC and subsequent 4-s $\mathrm{CS}+$ ) and near misses (occurring immediately after the IC ended). Systemic VTA-GABA activation did not alter perseverative, near miss, or spontaneous nosepokes compared to saline or GFP controls (Supplementary Figure S1B). Overall, the decrease in accuracy in Fig. 3a is therefore attributable to decreased rewarded nosepokes, rather than an increase in inappropriate responding when the reward was not available.

We also examined behavior in relation to the reward cup. After VTA-GABA activation, there was a Treatment $\times$ Virus interaction, due to a decrease in the number of reward cup entries during the session in hM3D rats after CNO pretreatment (Fig. $4 \mathrm{a}, F_{1,28}=6.04$, $p=0.02)$. VTA-GABA activation also decreased the mean number of rewards earned in the same rats $\left(F_{1,28}=89.77, p<0.0001\right)$, but not proportionally, such that saline treated and GFP control rats entered the reward cup 1.5-times/reward earned, while VTAGABA neuron activation increased this to $\sim 4$-times/reward (Treatment $\times$ Virus interaction, $F_{1,28}=6.44, p=0.017$ ). There were no changes in these metrics after intra-accumbal CNO administration. Together this suggests that activating VTA-GABA neurons disrupts responding during the $I C$, while some reward-seeking behaviors (e.g., cup entries) are preserved.

We next sought to determine whether the effects of VTA-GABA neuron activation occurred from the beginning of the session or required the rats to experience the reward. Rats normally respond to the first IC $(1.12 \pm 0.08$ th IC; $~ 33 \mathrm{~s}$ from start of session, Fig. $4 \mathrm{~b})$, but after VTA-GABA activation, the rats responded to a later IC $(1.94 \pm 0.33 \mathrm{rd}$ IC; $\sim 60 \mathrm{~s}$ from start of session, planned two-tailed Wilcoxon matched-pairs signed-rank test, $W=40, p=0.019$ ). Again, there was no effect on first IC responded to when VTAGABA terminals are activated by intra-NAc CNO infusions (Fig. 4b). Intra-accumbal cannula placements were also confirmed in Fig. 4b. These data indicate that the effects of VTA-GABA were present at the onset of the session, but again mesoaccumbal GABA does not have a role in these effects.

Given the apparent increase in cup entries when the reward was not available, we next sought to determine how VTA-GABA activation influenced the actions initiated after each IC (Fig. 4c). After saline, rats generally responded during an IC with a nosepoke and then reward cup entry to obtain the reward $\left(A_{+} \rightarrow B \rightarrow C\right.$; Fig. 1g). Occasionally, the reward cup was entered first, before nosepoking during an IC $\left(A_{+} \rightarrow C \rightarrow B \rightarrow C\right)$. After VTA-GABA activation, the number of $A_{+} \rightarrow B \rightarrow C$ rewarded action sequences significantly decreased (Fig. $4 C$, Treatment $\times$ Virus interaction, $F_{1,28}$ 


\section{A}
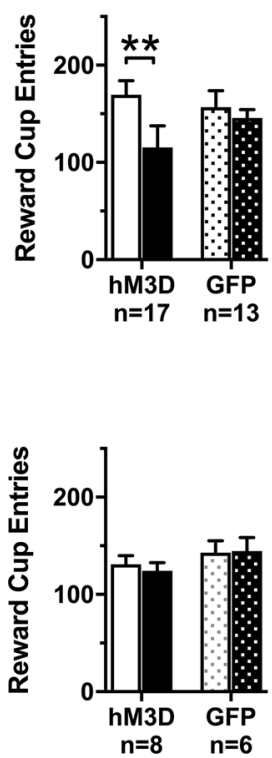

B Systemic

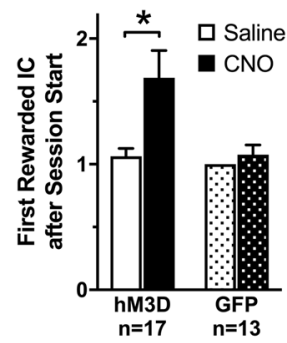

Systemic

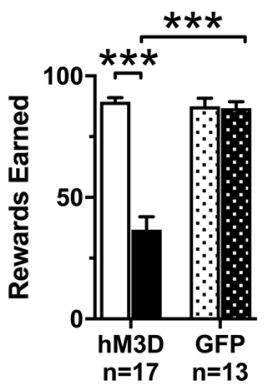

Intra-NAc
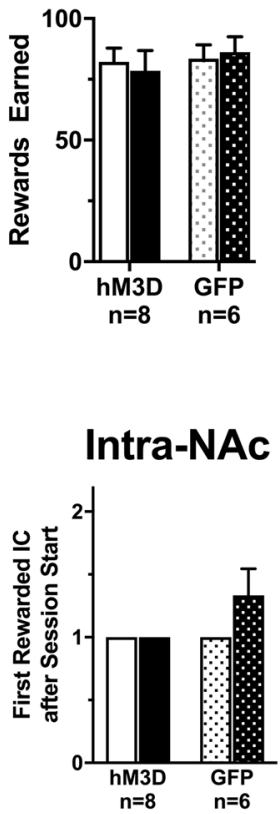

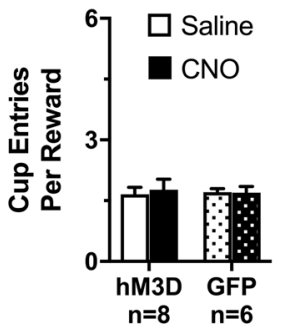

Cannula

Placements
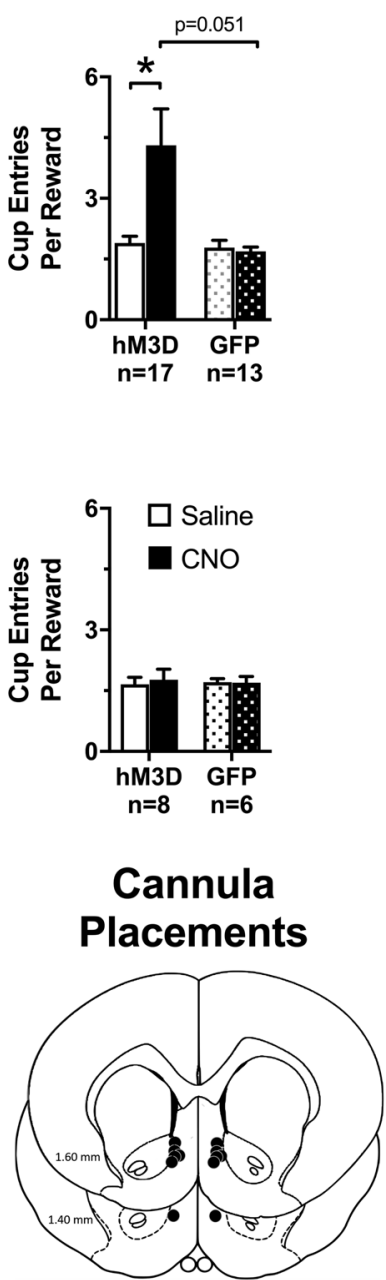

Unrewarded
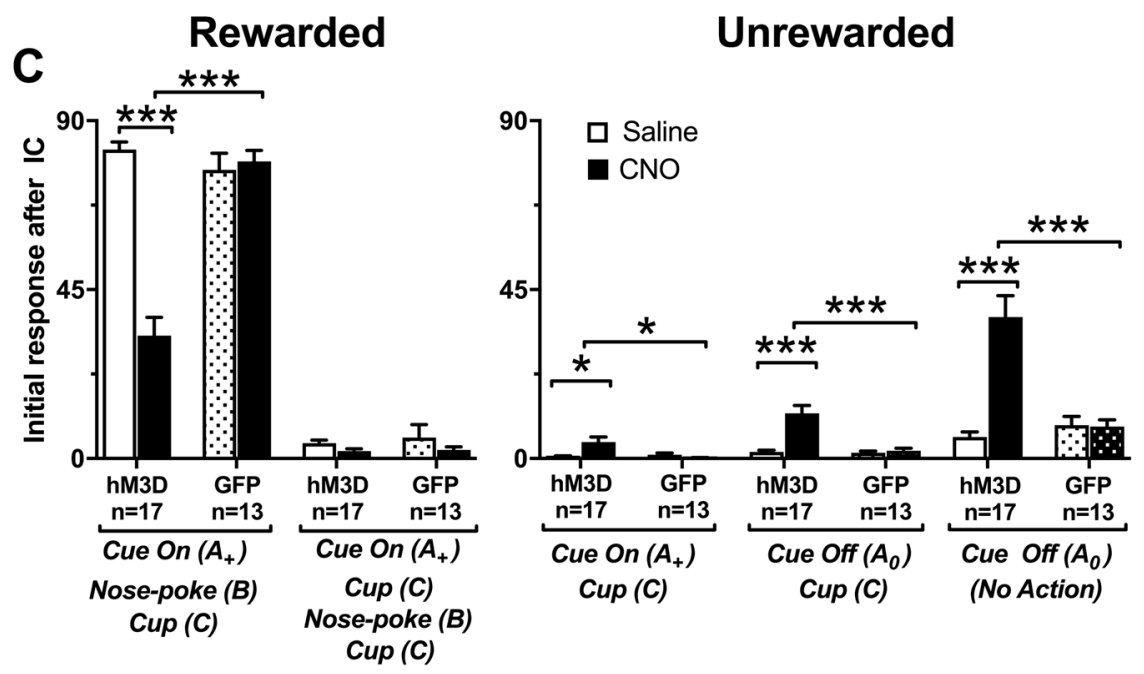

$=88.39, \quad p<0.0001)$. Effects on the other, less efficient $A_{+} \rightarrow C \rightarrow B \rightarrow C$ pattern were not significant. Unrewarded sequences simultaneously increased (Fig. 4C). Most frequently, rats stopped responding to the $\mathrm{IC}$ entirely and none of the action sequences examined were emitted $\left(A_{0}\right.$, Treatment $\times$ Virus interaction, $F_{1,28}=$
27.92, $p<0.0001)$, but reward cup entries between ICs also increased $\left(A_{0} \rightarrow C\right.$, Treatment $\times$ Virus interaction, $F_{1,28}=18.61, p<$ $0.0001)$, as well as reward cup entries during the IC $\left(A_{+} \rightarrow C\right.$, Treatment $\times$ Virus interaction, $F_{1,28}=9.10, p=0.005$ ). Thus, even though reward cup entries declined overall, unrewarded entries 
Fig. 4 VTA-GABA neuron activation disrupts instrumental performance while retaining some reward-seeking behaviors. All data are presented as mean \pm SEM. a Systemic but not intra-NAc CNO pretreatment significantly reduced reward cup entries during a session compared to saline control (top left), and systemic CNO pretreated rats achieved significantly less rewards than controls (top middle) so that reward cup entries per reward significantly increased after systemic CNO compared to saline (top right). There were no differences between intra-NAc pretreatments (bottom). b Systemic but not intra-NAc increased the mean first rewarded IC at the start of the session, determined by a planned two-tailed Wilcoxon matched-pairs signed-rank test. Note, in some sessions all rats responded to the first IC, thus some bars have no error bars. Far right panel indicate the location of the tip of the cannula in rats used for intra-NAc infusion experiments. c Systemic CNO pretreatment disrupted the appropriate behavioral response pattern to an IC. The number of initial rewarded action sequences after the presentation of the IC (left). Systemic CNO significantly decreased the number of IC $\left(A_{+}\right) \rightarrow$ nosepoke $(B) \rightarrow$ reward cup $(C)$ sequences. Conversely, CNO had no effect on the IC $\rightarrow$ reward cup $\rightarrow$ nosepoke $\rightarrow$ reward cup response. The number of unrewarded responses sequences after the IC (right) show that CNO significantly increased the number of reward cup entries during an IC presentation (i.e., bypassing a nosepoke: IC $\left(A_{+}\right) \rightarrow$ reward cup $(C)$ ), the number of reward cup entries when the IC was not present (end of IC $\left(A_{0}\right) \rightarrow \operatorname{reward}$ cup $(C)$ ) or when there was no detectable behavioral response to the IC $\left(A_{0}\right)$. Significant difference between treatment groups determined by a Holms-Sidak post hoc test. Asterisks represent significant differences in the statistical tests indicated $\left({ }^{*} p<0.05,{ }^{* *} p<0.01,{ }^{* * *} p<0.001\right)$
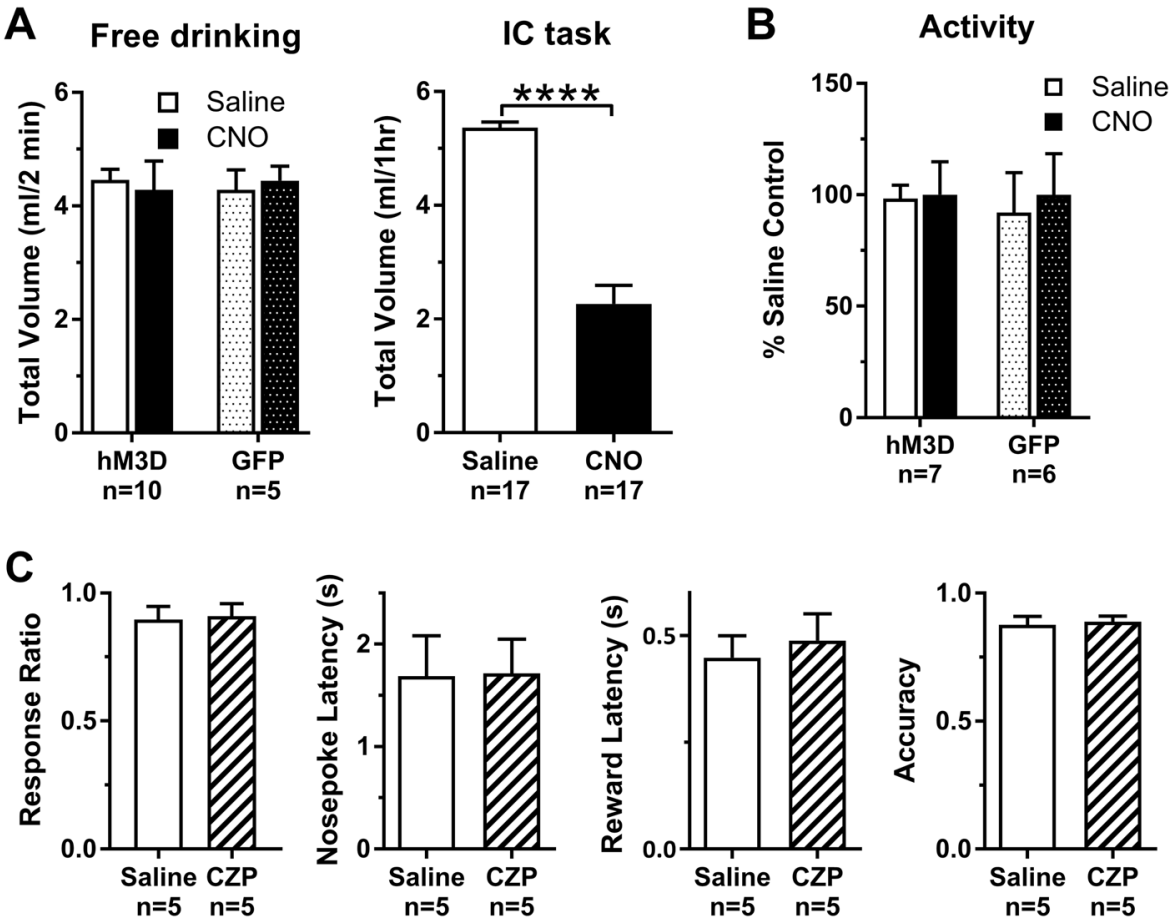

Fig. 5 VTA-GABA neuron activation does not impair sucrose consumption or locomotor behavior, and clozapine (CZP) has no effect on the incentive cue (IC) task. Data presented as mean \pm SEM. a Total volume of sucrose $(\mathrm{ml})$ during the first 2 min of a free-drinking session is equivalent to that obtained during an entire IC session; however, CNO decreased only the total volume consumed only during the IC task ( $t_{16}$ $=10.97, p<0.0001$ ) and not free-drinking. b Distance traveled (normalized as a percent of saline control) during a 20-min open-field locomotor test did not statistically differ between saline and CNO pretreatment. c Rats injected with a GFP sham virus into the VTA did not show a behavioral effect after systemic pretreatment with $0.3 \mathrm{mg} / \mathrm{kg}$ i.p. CZP across IC task metrics. Asterisks represent significant differences $(* * * 0<0.0001)$

increased. Rats engaged less in the most direct actions to obtain the reward $\left(A_{+} \rightarrow B \rightarrow C\right)$ and either ignored the IC $\left(A_{0}\right)$, sought the reward in absence of the IC $\left(A_{0} \rightarrow C\right)$, or erroneously entered the reward cup instead of the nosepoke during the IC $\left(A_{+} \rightarrow C\right)$.

Control experiments to determine whether VTA-GABA activation affects palatability, activity, or if metabolism of CNO to CZP impacts IC responding

To determine whether the effects of VTA-GABA stimulation could be attributed to changes in palatability of sucrose, we examined free drinking consumption. Under free drinking conditions, rats will drink in 2-min an equivalent amount of sucrose obtained during the entire 1-h IC task at baseline or after saline (Fig. 5a). VTA-GABA activation did not alter the amount of sucrose consumed in 2-min under free drinking conditions, even though the volume of sucrose consumed after VTA-GABA activation during the IC task decreased dramatically (Fig. 5b, two-tailed paired $t$-test, $\left.t_{16}=10.97, p<0.0001\right)$. These data indicate that the palatability of sucrose was unchanged and cannot account for the decreased responding to the ICs. In terms of generalized locomotor activity levels, the total distance traveled in an openfield after saline or VTA-GABA activation was not significantly altered via a Treatment $\times$ Virus interaction, again indicating that the primary results in the IC task did not arise from gross disruptions in overall locomotor activity. Finally, due to recent evidence that CNO is metabolized to CZP in vivo [32], we sought to determine whether any of the effects on the IC task were attributable to activity at receptors other than hM3D. We determined that an equivalent systemic CZP dose $(0.3 \mathrm{mg} / \mathrm{kg}$, i. p.) did not impact any of the metrics of the IC task (Fig. $5 \mathrm{C}$ ) in rats 
that do not express $\mathrm{hM} 3 \mathrm{D}$, indicating that metabolism to CZP is not contributing to the primary results of VTA-GABA activation in our earlier experiments.

\section{DISCUSSION}

This study produced three key findings. First, that activation of VTA-GABA neurons strongly reduced responding to cues predictive of reward availability by altering the cue's motivational salience. Second, targeted activation of VTA-GABA projections in the NAc did not impact this process. Third, this disruption was cue specific, and other, less efficient reward-seeking behaviors were preserved.

Pursuing and earning a reward in a dynamic environment is not a unitary process, but employs multiple reward-related systems with overlapping neural circuits [7, 33, 34]. Several reward processes have been ascribed to the mesolimbic circuitry, including attributing motivational properties to neutral stimuli (i.e., incentive salience [35]). It is therefore surprising that optogenetic studies have not found a strong and consistent effect of VTA-GABA activation on cue-induced reward-seeking. Indeed, these previous studies either could not distinguish between decreases in the motivational salience of the cue from decreases in reward consummatory behaviors [18], or determined that the primary effect of VTA-GABA activation is decreases in reward consumption, with no effect on non-instrumental responding to the reward-predictive cue [19]. We therefore chose to examine this question using a sophisticated and sensitive operant model, and found that VTA-GABA activation does in fact greatly inhibit instrumental responding to reward-predictive cues. Our results support earlier pharmacological evidence that in a task heavily dependent on incentive cues, VTA-GABA neurotransmission attenuates task performance [6]. We have extended these findings by demonstrating that VTA-GABA neuron activation alone is sufficient for producing this effect, establishing that the numerous mesoaccumbal GABA projections do not contribute, and by thoroughly characterizing the nature of the VTA-GABA disruption on ICinstrumental responding.

One proposed hallmark of changes in motivation underlying incentive motivational processes is that such changes should occur at the onset of the session, independent of exposure to the reward [35]. In our study, the rats demonstrated a CNO-induced decrease in motivation for the cue from the onset of the session, before having a chance to assess the value of the reward by consuming it. In addition, VTA-GABA activation did not change sucrose consumption during free-drinking, in that rats will readily consume a similar volume of sucrose compared to saline, and as earned during the IC task (Fig. 5a). Together, these results indicate VTA-GABA activation did not impact consummatory processes, such as reducing the palatability of the reward.

Our data indicate that VTA-GABA has a substantial effect on instrumental responding. The decrease in accuracy of nosepoking after VTA-GABA stimulation (Figs. 2a and 3a) may result from behavioral disinhibition and responding inappropriately to the cue, an effect also observed after pharmacological inactivation of the NAc in a similar task $[6,29]$. However, while VTA-GABA stimulation profoundly decreased responding during the IC (Fig. 3b), inappropriate perseverative, near-miss, and spontaneous nosepokes did not change (Supplementary Figure S1B). Thus, the change in accuracy resulted from a drop in responding to the cue, and not behavioral disinhibition leading to inappropriate nosepoking. The decreases in responding also likely do not result from a generalized suppression of locomotor activity (Fig. 5b). It should be noted that this IC task depends both on the ability of IC to elicit a motivated response, and the instrumental response itself. Therefore, it is not possible to completely dissociate the impact of VTA-GABA neuron activation on cue processing in the current behavior.
To determine whether VTA-GABA activation shifted motivational significance to the reward cup, we mapped the initial sequence of actions after each cue (Fig. 4c). Normally, the rats responded to the cue in the most efficient way (i.e., entering the reward cup immediately after nosepoking during the cue and rarely selected inefficient or incorrect responses). During VTAGABA activation, rats most commonly did not respond to the cues $\left(A_{0}\right.$, Fig. 4c). However, other inappropriate response patterns appeared, including bypassing the nosepoke for the reward-cup during the cue and reward-cup entries outside the presentation of the cue. Indeed, rats entered the reward cup $\sim 4$-times more often per earned reward (Fig. 4a). Thus, there was a decreased responding to the $\mathrm{IC}$ coupled with an increased sampling of the reward cup between ICs, suggesting the preservation in the motivation to seek the reward while disrupting responding to the IC directly. While it is possible that other motoric, perseverative or even stereotyped behaviors could account for disruption of IC responding, it seems unlikely given the lack of perseverative nosepoking, alterations in locomotor activity, or free-drinking behaviors.

Surprisingly, activation of VTA-GABA terminals in the NAc did not impact responding to reward-predictive cues in our behavioral task (Figs. 2b, 3b, 4a,b). Others have suggested that VTA-GABA neurons contribute to associative learning processes, which evaluate the outcome of a goal-directed action against a learned value $[17,18]$, and that VTA-GABA projections to the NAC may facilitate this process, possibly by disinhibiting local cholinergic interneurons [20]. However, VTA-GABA projection neurons send efferents to a wide range of other brain areas including the prefrontal cortex and amygdala $([7,15,16])$. VTA-GABA projections likely have unique influences on reward-seeking behavior, yet comparatively little is known about their contribution to motivated behaviors. In transgenic mice, optogenetic activation of VTA-GABA terminals in the NAC failed to alter sucrose consumption (i.e., licking, [19]) yet activation during training in a conditioned aversion paradigm enhanced predictive cue discrimination to an aversive stimulus [20]. The lack of effect from activating VTA-GABA accumbal projections in our well-trained rats may reflect that the motivational value of the cue remained constant throughout the session, leaving open the possibility that these projections have a role in associative learning. It should also be noted that systemic CNO would activate VTA-GABA interneurons and projection neurons in our study. Thus, the results reported here may be the result of either activating VTA-GABA interneurons, or activating a combination of interneurons and/or different VTA-GABA projection systems in the NAc or elsewhere. Future studies examining these learning processes may expose the role of the VTA-GABA projections to the NAC.

Notably, the validity of DREADD manipulations has recently been questioned, with a recent study showing that $\mathrm{CNO}$ is converted to CZP in vivo and is the primary metabolite to activate DREADDs [32]. Proper control studies are therefore important to interpret our results to determine whether activity at non-hM3D contributes to our effects. The dose of CNO used in these studies $(0.3 \mathrm{mg} / \mathrm{kg}$ i.p.) is low compared to other published studies [36], and well below the dose considered active in many DA related behaviors [37]. Importantly, this dose of CNO has no effect on any of the metrics of the IC task in sham treated rats that lack DREADDs (Figs. 2-5), nor does the $300 \mu \mathrm{mol}$ of CNO infused directly into the NAc (Figs. 2-4). We also observed a complete inhibition of firing of DA neurons when we applied $0.1 \mu \mathrm{M}$ of CNO in slices from hM3D expressing rats, and likewise this concentration appears to be much lower than other studies report (e.g., 5 $\mu \mathrm{M}$, see refs. $[38,39])$. We attribute our ability to successfully use this low dose to the high levels of transgene expression the AAV2/ 10 serotype affords. Alternatively, the VTA-GABA neurons may be more sensitive to $\mathrm{hM} 3 \mathrm{D}$ activation. Additionally, even if all of the CNO is converted to CZP, $0.3 \mathrm{mg} / \mathrm{kg}$ of CZP administered i.p. has 
been ineffective in altering other operant behaviors and likewise we showed no effect of this dose in our IC task (Fig. 5c). Thus we are confident that our results are not attributable to off-target effects of CNO.

In summary, we found that VTA-GABA neurons preferentially regulate processes contributing to the attribution of incentive salience, which are dissociable from other reward-seeking in consumption behaviors in a dynamic and robust operant model. VTA-GABA terminals do not disrupt incentive salience processes or responding to incentive cues, and future studies will need to determine what precise contributions these mesoaccumbal GABA projections have to the behavioral output of mesolimbic circuits.

\section{ACKNOWLEDGEMENTS}

We thank Dr. David C. Lyon for providing the GAD1 promoter used in these experiments. We thank Raquel Lima, Karie Chen, and Martin Leigh for technical assistance and Dr. Paul Meyer for very helpful comments on the manuscript. We also thank Dr. Wade Sigurdson and the University at Buffalo Confocal Microscopy Core for invaluable assistance. This research was supported by the State University of New York BRAIN Network of Excellence Postdoctoral Fellow program and T32 AA007583 (K.T.W.), the Whitehall Foundation 2017-08-43 (J.P.); 2017-12-98 (C.E.B.), as well as R01 AA024112 and R21 DA043190, (C.E.B.).

\section{ADDITIONAL INFORMATION}

Supplementary Information accompanies this paper at (https://doi.org/10.1038/ s41386-018-0097-6).

Competing interests: The authors declare no competing interests.

Publisher's note: Springer Nature remains neutral with regard to jurisdictional claims in published maps and institutional affiliations.

\section{REFERENCES}

1. Berridge $\mathrm{KC}$, Robinson TE. What is the role of dopamine in reward: hedonic impact, reward learning, or incentive salience? Brain Res Brain Res Rev. 1998;28:309-69.

2. Berridge $\mathrm{KC}$, Robinson TE. Liking, wanting, and the incentive-sensitization theory of addiction. Am Psychol. 2016;71:670-9.

3. Berridge KC, Robinson TE, Aldridge JW. Dissecting components of reward: 'liking', 'wanting', and learning. Curr Opin Pharmacol. 2009;9:65-73.

4. Hodge CW, Haraguchi M, Chappelle AM, Samson HH. Effects of ventral tegmental microinjections of the GABAA agonist muscimol on self-administration of ethanol and sucrose. Pharmacol Biochem Behav. 1996;53:971-7.

5. Miner P, Borkuhova Y, Shimonova L, Khaimov A, Bodnar RJ. GABA-A and GABA-B receptors mediate feeding elicited by the GABA-B agonist baclofen in the ventral tegmental area and nucleus accumbens shell in rats: reciprocal and regional interactions. Brain Res. 2010;1355:86-96.

6. Yun IA, Wakabayashi KT, Fields $\mathrm{HL}$, Nicola SM. The ventral tegmental area is required for the behavioral and nucleus accumbens neuronal firing responses to incentive cues. J Neurosci. 2004;24:2923-33.

7. Fields $\mathrm{HL}$, Hjelmstad GO, Margolis EB, Nicola SM. Ventral tegmental area neurons in learned appetitive behavior and positive reinforcement. Annu Rev Neurosci. 2007;30:289-316

8. Sesack SR, Grace AA. Cortico-Basal Ganglia reward network: microcircuitry. Neuropsychopharmacology. 2010;35:27-47

9. Watabe-Uchida M, Zhu L, Ogawa SK, Vamanrao A, Uchida N. Whole-brain mapping of direct inputs to midbrain dopamine neurons. Neuron. 2012;74:858-73.

10. Xia Y, Driscoll JR, Wilbrecht L, Margolis EB, Fields HL, Hjelmstad GO. Nucleus accumbens medium spiny neurons target non-dopaminergic neurons in the ventral tegmental area. J Neurosci. 2011;31:7811-6.

11. Zahm DS, Heimer L. Specificity in the efferent projections of the nucleus accumbens in the rat: comparison of the rostral pole projection patterns with those of the core and shell. J Comp Neurol. 1993;327:220-32.

12. Geisler S, Zahm DS. Afferents of the ventral tegmental area in the rat-anatomical substratum for integrative functions. J Comp Neurol. 2005;490:270-94.

13. Yau HJ, Wang DV, Tsou JH, Chuang YF, Chen BT, Deisseroth K, et al. Pontomesencephalic tegmental afferents to VTA non-dopamine neurons are necessary for appetitive pavlovian learning. Cell Rep. 2016;16:2699-710.
14. Creed MC, Ntamalti NR, Tan KR. VTA GABA neurons modulate specific learning behaviors through the control of dopamine and cholinergic systems. Front Behav Neurosci. 2014;8:8

15. Taylor SR, Badurek S, Dileone RJ, Nashmi R, Minichiello L, Picciotto MR. GABAergic and glutamatergic efferents of the mouse ventral tegmental area. J Comp Neurol. 2014:522:3308-34.

16. Van Bockstaele EJ, Pickel VM. GABA-containing neurons in the ventral tegmental area project to the nucleus accumbens in rat brain. Brain Res. 1995;682:215-21.

17. Cohen JY, Haesler S, Vong L, Lowell BB, Uchida N. Neuron-type-specific signals for reward and punishment in the ventral tegmental area. Nature. 2012;482:85-8.

18. Eshel N, Bukwich M, Rao V, Hemmelder V, Tian J, Uchida N. Arithmetic and local circuitry underlying dopamine prediction errors. Nature. 2015;525:243-6.

19. van Zessen R, Phillips JL, Budygin EA, Stuber GD. Activation of VTA GABA neurons disrupts reward consumption. Neuron. 2012;73:1184-94.

20. Brown MT, Tan KR, O'Connor EC, Nikonenko I, Muller D, Luscher C. Ventral tegmental area GABA projections pause accumbal cholinergic interneurons to enhance associative learning. Nature. 2012;492:452-6.

21. Liu YJ, Ehrengruber MU, Negwer M, Shao HJ, Cetin AH, Lyon DC. Tracing inputs to inhibitory or excitatory neurons of mouse and cat visual cortex with a targeted rabies virus. Curr Biol. 2013;23:1746-55.

22. Gompf HS, Budygin EA, Fuller PM, Bass CE. Targeted genetic manipulations of neuronal subtypes using promoter-specific combinatorial AAVs in wild-type animals. Front Behav Neurosci. 2015;9:152.

23. Illiano $P$, Bass CE, Fichera L, Mus L, Budygin EA, Sotnikova TD, et al. Recombinant adeno-associated virus-mediated rescue of function in a mouse model of Dopamine Transporter Deficiency Syndrome. Sci Rep. 2017:7:46280.

24. Mikhailova MA, Bass CE, Grinevich VP, Chappell AM, Deal AL, Bonin KD, et al. Optogenetically-induced tonic dopamine release from VTA-nucleus accumbens projections inhibits reward consummatory behaviors. Neuroscience. 2016;333:54-64.

25. Wakabayashi KT, Fields $\mathrm{HL}$, Nicola SM. Dissociation of the role of nucleus accumbens dopamine in responding to reward-predictive cues and waiting for reward. Behav Brain Res. 2004;154:19-30.

26. Anaclet C, Pedersen NP, Ferrari LL, Venner A, Bass CE, Arrigoni E, et al. Basal forebrain control of wakefulness and cortical rhythms. Nat Commun. 2015;6:8744.

27. Pedersen NP, Ferrari L, Venner A, Wang JL, Abbott SB, Vujovic N, et al. Supramammillary glutamate neurons are a key node of the arousal system. Nat Commun. 2017;8:1405

28. Mahler SV, Vazey EM, Beckley JT, Keistler CR, McGlinchey EM, Kaufling J, et al. Designer receptors show role for ventral pallidum input to ventral tegmental area in cocaine seeking. Nat Neurosci. 2014;17:577-85.

29. Ambroggi F, Ghazizadeh A, Nicola SM, Fields HL. Roles of nucleus accumbens core and shell in incentive-cue responding and behavioral inhibition. J Neurosci. 2011;31:6820-30.

30. Nair-Roberts RG, Chatelain-Badie SD, Benson E, White-Cooper H, Bolam JP, Ungless MA. Stereological estimates of dopaminergic, GABAergic and glutamatergic neurons in the ventral tegmental area, substantia nigra and retrorubral field in the rat. Neuroscience. 2008;152:1024-31.

31. Swanson LW. The projections of the ventral tegmental area and adjacent regions: a combined fluorescent retrograde tracer and immunofluorescence study in the rat. Brain Res Bull. 1982;9:321-53.

32. Gomez JL, Bonaventura J, Lesniak W, Mathews WB, Sysa-Shah P, Rodriguez LA, et al. Chemogenetics revealed: DREADD occupancy and activation via converted clozapine. Science. 2017;357:503-7

33. Kelley $A E$, Berridge $K C$. The neuroscience of natural rewards: relevance to addictive drugs. J Neurosci. 2002;22:3306-11.

34. Meyer PJ, King CP, Ferrario CR. Motivational processes underlying substance abuse disorder. Curr Top Behav Neurosci. 2016;27:473-506.

35. Berridge KC. From prediction error to incentive salience: mesolimbic computation of reward motivation. Eur J Neurosci. 2012;35:1124-43.

36. Trevitt JT, Lyons M, Aberman J, Carriero D, Finn M, Salamone JD. Effects of clozapine, thioridazine, risperidone and haloperidol on behavioral tests related to extramyramidal motor function. Psychopharmacology. 1997;132:74-81.

37. MacLaren DA, Browne RW, Shaw JK, Krishnan Radhakrishnan S, Khare P, Espana $\mathrm{RA}$, et al. Clozapine $\mathrm{N}$-oxide administration produces behavioral effects in longevans rats: implications for designing DREADD experiments. eNeuro. 2016;3: e0219_16:016 1-14.

38. López AJ, Kramár E, Matheos DP, White AO, Kwapis J, Vogel-Ciernia A, et al. Promoter-specific effects of DREADD modulation on hippocampal synaptic plasticity and memory formation. J Neurosci. 2016;36:3588-99.

39. Buchta WC, Mahler SV, Harlan B, Aston-Jones GS, Riegel AC. Dopamine terminals from the ventral tegmental area gate intrinsic inhibition in the prefrontal cortex. Physiol Rep. 2017;5:e13198. 